\title{
Transcriptomics of plants in the Chernobyl exclusion zone: a step closer to understand the adaptation to chronic radiation exposure
}

\author{
Volkova P.Yu. ${ }^{1 *}$, Duarte G.T. ${ }^{2}$, Podlutskii M.S. ${ }^{1}$ \\ ${ }^{1}$ Russian Institute of Radiology and Agroecology, Obninsk, Russia \\ ${ }^{2}$ Max Plank Institute of Molecular Plant Physiology, Potsdam, Germany \\ *email:volkova.obninsk@gmail.com
}

The Chernobyl exclusion zone (CEZ) provides unique opportunities for investigation the effects of long-term chronic radiation exposure on plant populations. Plants belonging to different genera can significantly differ in sensitivity to acute and chronic radiation exposure. Field sampling was performed in the CEZ to investigate the transcriptional profiles of herbaceous and tree species growing in the conditions of chronic radiation exposure. The transcriptomic outputs for a radiosensitive tree species Pinus sylvestris and a radioresistant herbaceous species Capsella bursa-pastoris were compared, suggesting an important role of histones, chaperons, and control of transposable elements in plant adaptation to chronic exposure to radionuclides. The seeds of Arabidopsis thaliana plants sampled in the CEZ were subjected to acute high-dose irradiation, and RNA-seq was performed to identify differentially expressed genes triggered by irradiation. The response profiles of plants to acute and chronic radiation exposure were compared, revealing common and distant trends in transcriptomic responses to different modes of exposure.

Acknowledgements: The research has been supported by Russian Science Foundation, project No. 20-74-10004. 